\title{
Two Results on Intersection Graphs of Polygons
}

\author{
Jan Kratochvíl and Martin Pergel \\ Department of Applied Mathematics and \\ Institute for Theoretical Computer Science ${ }^{\star}$ \\ Charles University \\ Malostranské nám. 25 \\ 11800 Praha 1 \\ Czech Republic \\ $\{$ honza,perm\}@kam.mff.cuni.cz
}

\begin{abstract}
Intersection graphs of convex polygons inscribed to a circle, so called polygon-circle graphs, generalize several well studied classes of graphs, e.g., interval graphs, circle graphs, circular-arc graphs and chordal graphs. We consider the question how complicated need to be the polygons in a polygon-circle representation of a graph.

Let $\operatorname{cmp}(n)$ denote the minimum $k$ such that every polygon-circle graph on $n$ vertices is the intersection graph of $k$-gons inscribed to the circle. We prove that $\operatorname{cmp}(n)=n-\log _{2} n+o\left(\log _{2} n\right)$ by showing that for every positive constant $c<1, \operatorname{cmp}(n) \leq n-c \log n$ for every sufficiently large $n$, and by providing an explicit construction of polygon-circle graphs on $n$ vertices which are not representable by polygons with less than $n-\log n-2 \log \log n$ corners. We also show that recognizing intersection graphs of $k$-gons inscribed in a circle is an NP-complete problem for every fixed $k \geq 3$.
\end{abstract}

\section{Introduction}

Intersection graphs of geometric objects, namely in the plane, are intensively studied both for their practical motivations and for interesting structural and algorithmic properties. Many hard (NP-complete in general) optimization problems become polynomially solvable when restricted to various classes of intersection graphs. Probably the oldest and simplest of these are interval graphs, intersection graphs of intervals on a line [4], whose structure is well understood, they are recognizable in linear time, and for which problems like clique, independent set, dominating set, chromatic number and many more are tractable. On the other end of the spectrum are string graphs, intersection graphs of arc-connected sets in the plane, which are hard to recognize [8], and whose recognition was only recently shown decidable [12]15] and then even more surprisingly in NP [16].

Geometric intersection graphs also provide a special kind of graph visualization. Vertices are represented as geometric objects and adjacencies are visualized by nonempty intersections. Overlapping regions are illustrative and enhance the visual understanding of the represented graph. String graphs are closely related to another graph drawing invariant, the crossing number, i.e., the minimum number of edge crossings in a drawing

\footnotetext{
${ }^{\star}$ Project LN00A056 supported by the Ministry of Education of the Czech Republic.
} 
of a graph. Abstract topological graphs (cf. [10]) are graphs with specified pairs of edges which are allowed to cross in a feasible drawing. In this setting it may happen that multiple edge crossings occur in every feasible drawing, and an abstract topological graph may actually require exponential number of crossing points [11]. The crossing number of abstract topological graphs is closely related to the number of crossing points in representations of string graphs as intersection graphs of curves, and in fact in this way the decidability and NP-membership of string graphs were tackled.

Several well studied classes of graphs which directly generalize interval graphs involve geometric objects bound to a circle. Among these are circle graphs, intersection graphs of chords of a circle, and circular arc graphs, intersection graphs of intervals on the circle. Both these classes are recognizable in polynomial time (cf. [1.3 13] for circle graphs, [172] for circular arc graphs). A common generalization of these two are polygon-circle graphs, intersection graphs of convex polygons inscribed to the circle. This class was first suggested by M. Fellows [personal communication with the first author] in 1988, when it was pointed out that this class of graphs is closed under taking induced minors. Under a different name of spider graphs, polygon-circle graphs appeared in [7], where a polynomial time recognition algorithm was announced. Somewhat surprisingly, the algorithm was never published, and this fact creates certain doubts about its correctness. A structural property of graph classes which many intersection graphs possess is near-perfectness in the sense of Gyarfás [5]. A graph class is near-perfect if the chromatic number of each of its graphs is bounded by a function of the clique number of the graph. (For perfect graphs this function is identity, and from here the notion originates.) Polygon-circle graphs are near-perfect as shown in [9], and the bound presented therein improved the up to that date best bound for circle graphs and has not been improved since.

In the current paper we pay closer attention to the question of how complicated should be the polygons representing the vertices of a polygon-circle graph with $n$ vertices. One can easily see that $n$-gons always suffice (hence no exponential surprises as in the case of string graphs), which means that polygon-circle graph recognition is definitely in NP. It is conceivable, however, that polygons with less corners would suffice. To be able to precisely formulate this question, we define the complicacy of a graph $G$ as the minimum $k$ such that $G$ is the intersection graph of convex $k$-gons inscribed to a circle, and we denote this invariant by $\operatorname{cmp}(G)$ (we set $\operatorname{cmp}(G)=\infty$ if $G$ is not a polygon-circle graph). We further define $\operatorname{cmp}(n)$ to be the maximum of $\operatorname{cmp}(G)$ over all polygoncircle graphs with $n$ vertices. The main result in this direction is the following (here and throughout the paper, all logarithms are base 2):

Theorem 1. We have $\operatorname{cmp}(n)=n-\log n+o(\log n)$.

The lower and upper bounds are proved separately in the next two sections. In the last section we consider the computational complexity of determining the complicacy of a graph with the following result:

Theorem 2. For every fixed finite $k \geq 3$, it is $N P$-complete to decide whether $\operatorname{cmp}(G) \leq$ $k$ holds for an input graph $G$.

This result, which answers an open problem listed at J. Spinrad's web page [14], is not in contradiction with the announced algorithm of Koebe for recognition of polygon- 
circle graphs (i.e., deciding $\operatorname{cmp}(G)<\infty$ ), but it definitely sheds new light on the problem of recognizing polygon-circle graphs. Note also that for $k=2, \operatorname{cmp}(G) \leq 2$ if and only if $G$ is a circle graph, a polynomially decidable question.

\section{Technical Notions and Observations}

Throughout the paper we use small letters as $a, b, u, v, \ldots$ for vertices of the graph under consideration, and if $R$ is a representation by polygons, $R_{v}$ denotes the polygon representing vertex $v$. However, in figures, to avoid multiple subscripts, we will usually omit the symbol $R$. We assume that the bounding circle is fixed and whenever we speak about polygons, we automatically assume that the polygons are convex and have all corners placed on the circle.

If $P$ is a polygon, then the connected parts obtained from the bounding circle by deleting the corners of $P$ are referred to as the $P$-segments. If two polygons represent nonadjacent vertices, they must be disjoint and hence all corners of one of them lie within the same segment determined by the other one, and vice versa. In the following technical definition we assume that all polygons under consideration are disjoint.

Definition 1. We say that polygon $P$ blocks polygon $Q$ from polygon $S$ if the corners of $Q$ lie in a different $P$-segment than the corners of $S$. If a set $\mathcal{S}$ of polygons is such that none of them blocks any other two polygons from each other, we say that the polygons are positioned around the circle.

See Figure 1 for an illustrative example of blocking polygons, and a set of polygons positioned around the circle. Next we make the first simple but useful observation.
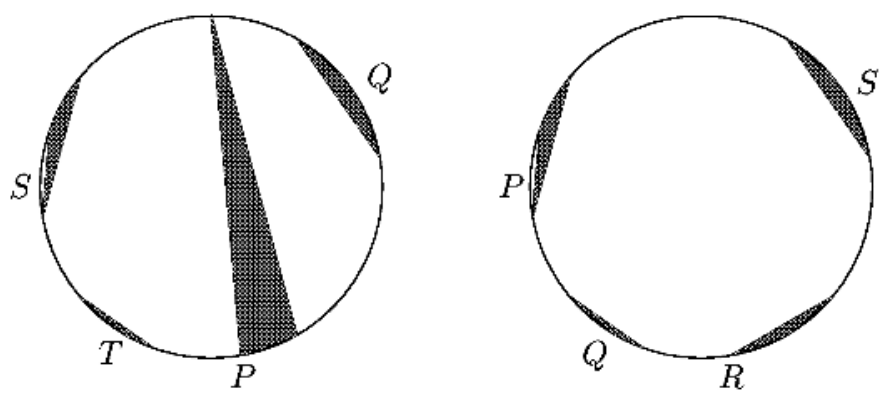

Fig. 1. In the left, polygon $P$ blocks polygon $Q$ from polygons $S$ and $T$, in the right, all four polygons are positioned around the circle.

Proposition 1. In any representation $R$ of the cycle $C_{2 k}$ with $2 k$ vertices $u_{1}, u_{2}, \ldots, u_{2 k}$, the polygons $R_{u_{2 i}}: i=1,2, \ldots, k$ are positioned around the circle. If $W_{k}^{\prime}$ is the graph obtained from $C_{2 k}$ by adding a vertex $v$ adjacent to $u_{2 i}, i=1,2, \ldots, k$ (i.e., $W_{k}^{\prime}$ is the wheel $W_{k}$ with each rim edge subdivided), then $R_{v}$ has at least $k$ corners and $\operatorname{cmp}\left(W_{k}^{\prime}\right) \geq k$ (in fact, the complicacy of $W_{k}^{\prime}$ equals $k$ ). 

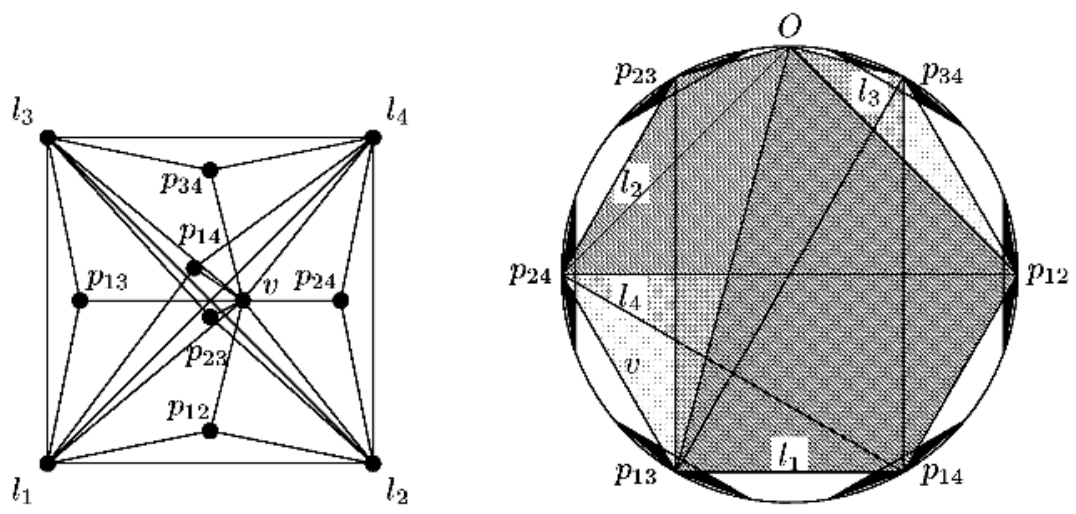

Fig. 2. An example of the lower bound construction for $\ell=1$ and $n=11$. We write simply $p_{13}$ for $p_{\{1,3\}}$ etc.

Proof. For any three (even indexed) vertices $u_{2 i}, u_{2 j}, u_{2 h}$ of $C_{2 k}$, there exists a path connecting $u_{2 j}$ and $u_{2 h}$ which does not contain any neighbor of $u_{2 i}$. The union of the polygons representing vertices of this path is a connected subset of the disk bounded by the base circle, and hence (by the Jorda curve theorem) $R_{u_{2 j}}$ must not be blocked from $R_{u_{2 h}}$ by $R_{u_{2 i}}$.

For the wheel graph $W_{k}^{\prime}$, it follows that $R_{v}$ must have at least $k$ corners, to intersect all $R_{u_{2 i}}, i=1,2, \ldots, k$.

\section{Complicacy of Representations - The Lower Bound}

Theorem 3. For $n$ large enough, we have $\operatorname{cmp}(n) \geq n-\log n-2 \log \log n$.

Proof. The proof is by constructing graphs of large complicacy by an explicit construction. Suppose $n$ is large enough (how large will follow from the calculations in the proof). Let $\ell$ be the uniquely defined integer such that

$$
1+2 \ell+\left(\begin{array}{c}
2 \ell \\
\ell
\end{array}\right)<n \leq 1+2(\ell+1)+\left(\begin{array}{c}
2 \ell+2 \\
\ell+1
\end{array}\right) .
$$

We construct the graph $G=(V, E)$ with vertex set

$$
V=\{v\} \cup L \cup P,
$$

where vertex $v$ is adjacent to all other vertices, $L$ is a clique of size $2 \ell+2$, and $P$ is an independent set whose vertices are indexed by distinct $(\ell+1)$-element subsets of $L$. These indices will determine the adjacencies between vertices of $P$ and $L$ as follows. If $p_{a} \in P$ (with $a \subset L,|a|=\ell+1$ ), we make $p_{a}$ adjacent to all $x \in a$ and to no other vertices of $L$. (Note that $G$ is not determined uniquely, it depends on the choice of the sets used for indexing the vertices from $P$. Any choice of distinct indices works.) 
We claim that $G$ is a polygon-circle graph and that $\operatorname{cmp}(G) \geq|P|$. For the first part of the claim, choose a point $O$ on the circle and position the polygons corresponding to the vertices of $P$ around the circle (so that they do not block $O$ from one another). Choose one corner of each of them as its reference point and represent each vertex $x$ of $L$ by the convex hull of the reference points of the vertices of $P$ adjacent to $x$ and of $O$. By making $O$ a corner of each $R_{x}$ we guarantee that every two polygons $R_{u}, R_{w}, u, w \in L$ intersect ( $L$ is a clique). Finally $R_{v}$ will be the convex hull of $O$ and the reference points of all polygons representing vertices of $P$. (Note that the auxiliary point $O$ may not be necessary, e.g., in the case when for every two vertices in $L, P$ contains a vertex adjacent to both of them.)

To argue that $\operatorname{cmp}(G)$ is large, consider an optimal representation $R$ of $G$. Note first that for $n>5$ we have $\ell \geq 1$ and hence $\left(\begin{array}{c}2 \ell \\ \ell\end{array}\right) \geq 2$. This means that $n>2 \ell+3$ and indeed $G$ contains all vertices of $L$.

The key observation is that the polygons $R_{p}, p \in P$ must be positioned around the circle. For suppose this is not the case, say $R_{p_{b}}$ blocks $R_{p_{a}}$ from $R_{p_{c}}$ for some $a, b, c \subset L$. Since these subsets are different but of equal size, there must be an $x \in a \backslash b$ and a $y \in c \backslash b$. By the definition of $G, R_{x}$ intersects $R_{a}$ and $R_{y}$ intersects $R_{c}$, but none of $R_{x}, R_{y}$ intersects $R_{b}$. But that means that $R_{b}$ blocks $R_{x}$ from $R_{y}$ and these two polygons cannot intersect each other (though $x$ and $y$ belong to the clique $L$ ).

To intersect all polygons representing vertices of $P, R_{v}$ must have at least $|P|$ corners, and hence $\operatorname{cmp}(G) \geq|P|=n-2 \ell-3$. The rest is a simple calculation.

Assume for contradiction that $\ell>\frac{\log n}{2}+\log \log n-\frac{3}{2}$. Then

$$
n>\left(\begin{array}{c}
2 \ell \\
\ell
\end{array}\right)>\frac{2^{2 \ell}}{2 \ell+1}>\frac{n \log ^{2} n}{8(\log n+2 \log \log n-2)}>n
$$

for large enough $n$ ( ince $\left.\lim _{n \rightarrow \infty} \frac{\log ^{2} n}{8(\log n+2 \log \log n-2)}=\infty\right)$, a contradiction. Therefore (for every large enough $n$ ), $\ell \leq \frac{\log n}{2}+\log \log n-\frac{3}{2}$ and

$$
\operatorname{cmp}(G) \geq|P|=n-2 \ell-3 \geq n-\log n-2 \log \log n
$$

as claimed.

\section{Complicacy of Representations - The Upper Bound}

Theorem 4. For every positive constant $c<1$, there exists an $n_{0}$ such that $\operatorname{cmp}(n) \leq$ $n-c \log n$ for every $n>n_{0}$.

Proof. Let $G=(V, E)$ be a graph on $n$ vertices and let $\operatorname{cmp}(G)=k \geq 4$. Consider a polygon-circle representation $R$ of $G$ such that no two polygons share a corner and such that every polygon has at most $k$ corners (this can be always achieved by splitting the corners). Let our representation have the minimum total number of corners among all such representations. Choose a vertex $v \in V$ such that $R_{v}$ has $k$ corners, and denote its corners $v^{1}, v^{2}, \ldots, v^{k}$ as they appear clockwise around the circle.

Based on this representation $R$, define $A$ to be the set of vertices $x$ such that $R_{x}$ has all corners within two consecutive $R_{v}$-segments, i.e., such that $R_{x}$ intersects $R_{v}$ only in 


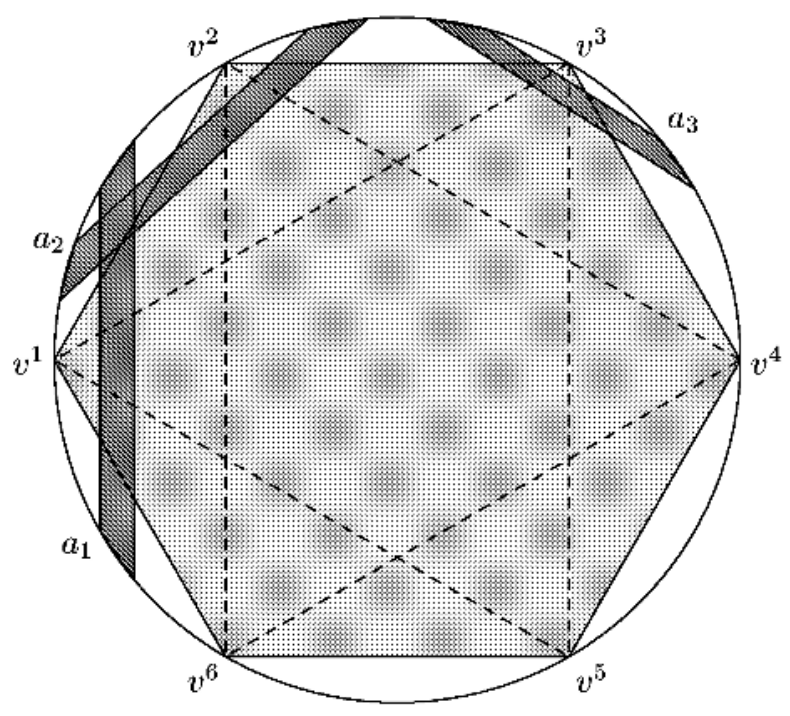

Fig. 3. Illustration to the choice of representatives of index classes.

the triangle $v^{i-1} v^{i} v^{i+1}$, for some $i$. For $x \in A$, denote this $i$ by $j(x)$ and call it the index of $x$. We can see that for every $i$, there exists an $x \in A$ of index $i$. For if such a vertex did not exist for some $i$, we could reduce the number of corners in the representation by deleting the triangle $v^{i-1} v^{i} v^{i+1}$ from $R_{v}$, contradicting the choice of $R$.

Now assume that $R^{\prime}$ minimizes $\sum_{x \in A} j(x)$ among all representations with the same central polygon $R_{v}$, with the same total number of corners and with the same set $A$ (when defined as above) as $R$. For the sake of simplicity we call $R^{\prime}$ again just $R$.

For every $i$, choose one vertex as a representative of the vertices of index $i$, and call it $a_{i}$. As argued above, $a_{i}$ is well defined for every $i$. The intersection graph of the polygons $R_{a_{i}}, i=1,2, \ldots, k$ is either a cycle or a disjoint union of paths, since $a_{i}$ can only intersect $a_{i-1}$ and/or $a_{i+1}$. We denote $A_{1}$ the set of those $a_{i}$ that have no neighbors among the other $a_{h}$ 's, and $A_{2}=\left\{a_{1}, a_{2}, \ldots, a_{k}\right\} \backslash A_{1}$. We then denote $B=(V \backslash\{v\}) \backslash\left(A_{1} \cup A_{2}\right)$ and $B_{1}$ the set those vertices of $B$ which are adjacent to at least one vertex of $A_{1}$.

Next we claim that every two vertices of $A_{1}$ have different sets of neighbors. For suppose that $\left\{x \mid x a_{i} \in E\right\}=\left\{x \mid x a_{j} \in E\right\}$ for some $j<i$. Then the polygons representing the neighbors of $a_{i}$ intersect one side of $R_{a_{j}}$, and hence both $R_{a_{i}}$ and $R_{a_{j}}$ could be replaced by two parallel chords (digons) placed close enough to this side. This would result in a new representation of the same graph, with the same position of $R_{v}$ and the same set $A$, but with a strictly smaller sum of the indices of the vertices of $A$, contradicting the choice of $R$. See an illustrative example in Figure 4

The immediate but very important consequence is that

$$
\left|A_{1}\right| \leq 2^{\left|B_{1}\right|}
$$



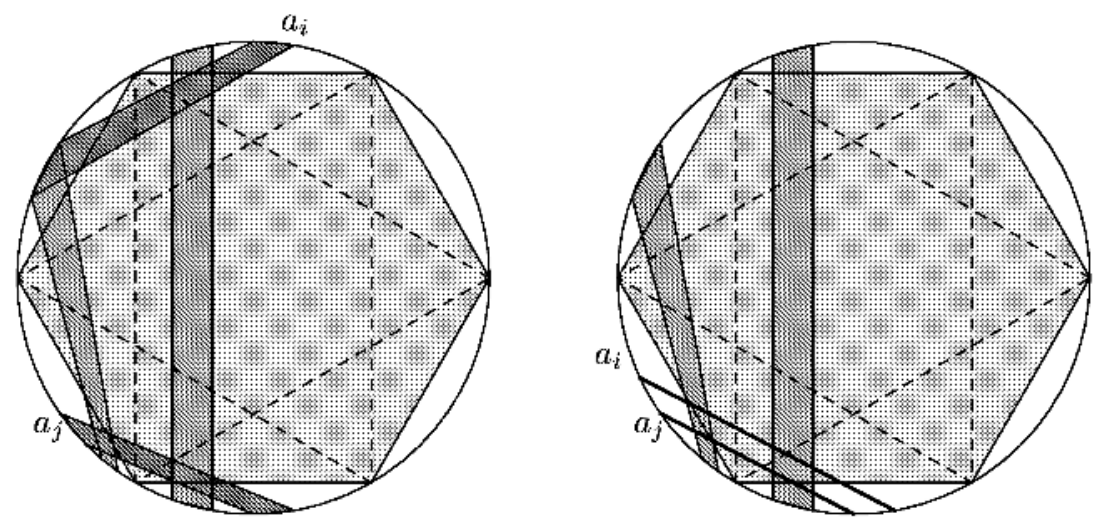

Fig. 4. Illustration to reducing the sum of indices of the $A$ vertices.

since $v$ is a common neighbor of all vertices in $A_{1}$, and on the other hand no vertex of $A_{1}$ is adjacent to any other vertex of $A_{1} \cup A_{2}$.
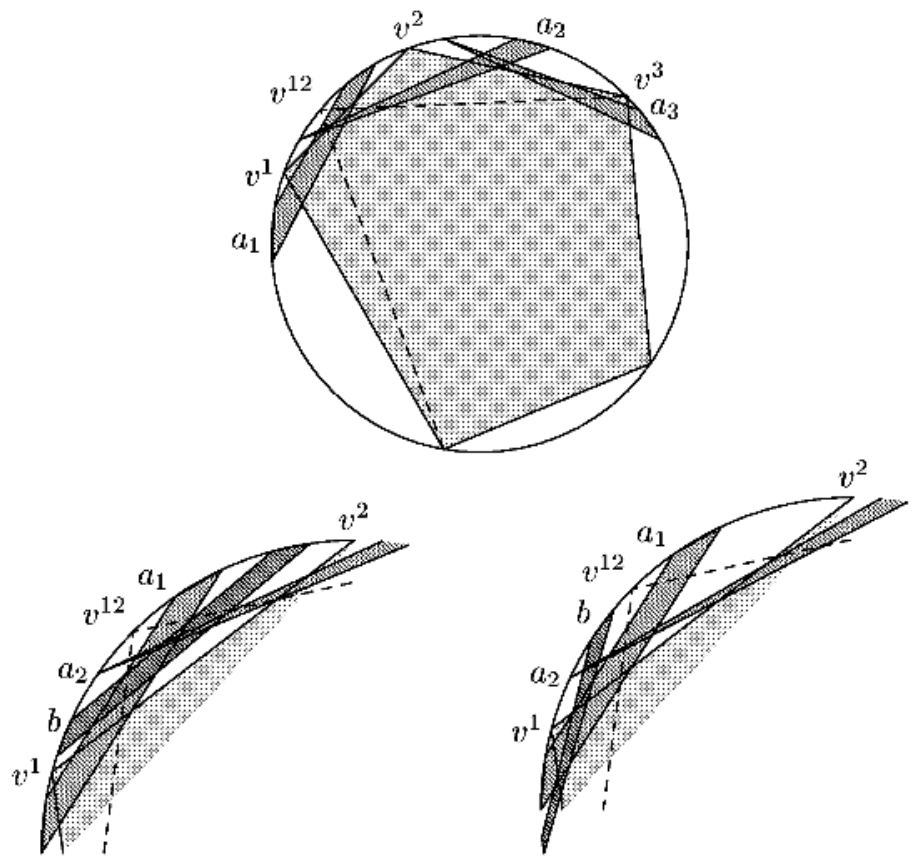

Fig. 5. Illustration to the construction of $B_{2}$, the blocking set for $A_{2}$. 
Finally we attend to vertices of $A_{2}$. Consider the example in Figure 5. If $R_{a_{1}}$ and $R_{a_{2}}$ intersect, they must intersect in the area bounded by the $v^{1} v^{2}$ side of $R_{v}$ and the corresponding $R_{v}$-segment. Choose a point $v^{12}$ in this segment between the leftmost corner of $a_{2}$ and the rightmost corner of $a_{1}$. If we now replaced the central polygon $R_{v}$ by the convex hull of $v^{12}, v^{3}, \ldots, v^{k}$ (i.e., the corners $v^{1}$ and $v^{2}$ are replaced by $v^{12}$ ), the new $R_{v}$ (denoted by dashed lines in the figure) would still intersect both $R_{a_{1}}$ and $R_{a_{2}}$, but the total number of corners would decrease. Since this is impossible by the choice of $R$, the new collection of polygons does not represent $G$ anymore. There are only two possible reasons. Either the new $R_{v}$ intersects a polygon $R_{b}$ which is not supposed to be intersected (as in Figure 5 bottom left), or the new $R_{v}$ loses intersection with some $R_{b}$ that it previously crossed (as in Figure 5 bottom right). In the former case, $R_{b}$ would lie fully within the segment $v^{1} v^{2}$, in the latter one, $b \in A$ and its index is $j(b)=1$ (or, symmetrically, $j(b)=2$ ). In any case, we choose one such $b$ and call it a witness for $(1,2)$. Similarly, we choose a witness for every $(i, i+1)$ such that $R_{a_{i}} \cap R_{a_{i+1}} \neq \emptyset$, and denote by $B_{2}$ the set of witnesses constructed in this way. By the construction, $B_{2} \subset B$. It may happen, though, that one vertex of $B_{2}$ is a witness for two pairs (a witness of the latter type and index $i$ could have been chosen both for the pair $(i-1, i)$ and for $(i, i+1))$. Given the fact that a path of $t$ consecutive vertices from $A_{2}$ would involve $t-1$ pairs, such a path would give rise to at least $\frac{t-1}{2}$ distinct witnesses, and we obtain the following inequality:

$$
\left|A_{2}\right| \leq 3\left|B_{2}\right|
$$

(Note here, that $B_{1}$ and $B_{2}$ are not necessarily disjoint.)

Now we summarize

$$
k=\operatorname{cmp}(G)=\left|A_{1}\right|+\left|A_{2}\right| \leq 2^{\left|B_{1}\right|}+3\left|B_{2}\right| \leq 2^{n-k}+3(n-k)
$$

(the last inequality follows from the fact that $A_{1} \cup A_{2} \cup B_{1} \subset V$ and $A_{1} \cup A_{2} \cup B_{2} \subset V$, while in each case the three summands are pairwise disjoint), and hence

$$
2^{n} \geq 2^{k}(4 k-3 n)
$$

For $k>n-c \log n(0<c<1)$, we would get

$$
2^{n}>2^{n-c \log n}(4(n-c \log n)-3 n)=2^{n} \frac{n-4 c \log n}{n^{c}}>2^{n}
$$

for every $n>n_{0}$ for some $n_{0}$, as $\lim _{n \rightarrow \infty} \frac{n}{n^{c}}=\infty$ and $\lim _{n \rightarrow \infty} \frac{4 c \log n}{n^{c}}=0$. Which is the final long worked for contradiction.

The proof of Theorem $\square$ is now at hand. Consider the difference $f(n)=\operatorname{cmp}(n)-$ $n+\log n$. On one hand, Theorem 3 states that $f(n) \geq-2 \log \log n=o(\log n)$. On the other hand, Thereom 4 yields that for every $\varepsilon>0, f(n)<\varepsilon \log n$ for every large enough $n$ (by setting $c=1-\varepsilon$ ), and hence $f(n)=o(\log n)$. 


\section{Computational Complexity}

We first restate Thereom 2 in a slightly stronger form:

Theorem 5. For every fixed $k \geq 3$, it is $N P$-complete to decide if $\operatorname{cmp}(G) \leq k$ for an input graph $G$, even if $G$ is promised to have complicacy at most $k+1$.

Proof. We reduce from the following hypergraph coloring problem: Given a set $\mathcal{T}$ of triples over a base set $X$, decide if the elements of $X$ can be colored by three colors so that every triple $T \in \mathcal{T}$ receives all three colors. This problem is NP-complete as it contains the 3-edge colorability of cubic graphs as a subproblem, the latter being NPcomplete by Holyer [6]. (By resctricting to 3-edge colorability of bridgeless graphs, we may assume that $X$ is colorable by 2 colors so that no triple $T \in \mathcal{T}$ is monochromatic.)

Given such a $\mathcal{T}$, we construct a graph $G_{\mathcal{T}}=(V, E)$ as follows. The vertex set will consist of a cycle of length $2 k$ on vertices $u_{1}, u_{2}, \ldots, u_{2 k}$, a pair of vertices $a_{x}, b_{x}$ for every $x \in X$, and a vertex $c_{T}$ for each triple $T \in \mathcal{T}$. The $c$ vertices form a clique and each of them is adjacent to all $u$ vertices with even subscripts, to all $b$ vertices, and to those $a$ vertices which correspond to $x$ 's belonging to the particular triple. The $b$ vertices are further adjacent to their corresponding $a$ vertices and to $u_{2}, u_{4}, u_{6}$. Formally

$$
\begin{gathered}
V=\left\{u_{i} \mid i=1,2, \ldots, 2 k\right\} \cup\left\{a_{x}, b_{x} \mid x \in X\right\} \cup\left\{c_{T} \mid T \in \mathcal{T}\right\} \\
E=\left\{u_{1} u_{2}, u_{2} u_{3}, \ldots, u_{2 k} u_{1}\right\} \cup\left\{a_{x} b_{x} \mid x \in X\right\} \cup\left\{b_{x} u_{i} \mid x \in X, i=2,4,6\right\} \\
\cup\left\{b_{x} b_{y} \mid x, y \in X\right\} \cup\left\{c_{T} a_{x} \mid x \in T \in \mathcal{T}\right\} \cup\left\{c_{T} b_{x} \mid T \in \mathcal{T}, x \in X\right\} \\
\cup\left\{c_{T} c_{S} \mid T, S \in \mathcal{T}\right\} \cup\left\{c_{T} u_{i} \mid T \in \mathcal{T}, i=2,4,6, \ldots, 2 k\right\} .
\end{gathered}
$$

We claim that $G_{\mathcal{T}}$ is always a PC graph of complicacy at most $k+1$, and its complicacy is (at most) $k$ if and only if $\mathcal{T}$ allows a coloring as desired.

Let $R$ be a PC representation of $G_{\mathcal{T}}$. We first note that all polygons $R_{a_{x}}, x \in X$ must be positioned around the circle. For if one of them would block another two from each other, some $R_{x}$ would block some $R_{y}$ from $R_{u_{2}}$ and the auxiliary polygon $R_{b_{y}}$ would not be able to intersect both $R_{y}$ and $R_{u_{2}}$.

As observed in Proposition 11 the polygons $R_{u_{2 i}}, i=1,2, \ldots, k$ are positioned around the circle, and only these vertices of the cycle are intersected by $R_{c_{T}}$ 's. Since the polygons representing the $b$ 's intersect (only) $R_{u_{2}}, R_{u_{4}}$ and $R_{u_{6}}$, all the polygons representing the $a$ vertices must lie in the segments determined by $R_{u_{2}}, R_{u_{4}}$ and $R_{u_{6}}$. So color an element $x \in X$ by color $i$ if $R_{a_{x}}$ is blocked by $R_{u_{i}}$ from the other $R_{u_{j}}$, $i, j=2,4,6$. We claim that this is a good coloring. For if a triple $T \in \mathcal{T}$ contained two vertices of the same color, say $x$ and $y$ of color 2 , the polygon $R_{c_{T}}$ would need $k-1$ corners for intersections with $R_{u_{i}}, i=4,6, \ldots, 2 k$, plus at least two more corners for intersections with $R_{x}$ and $R_{y}$, that is at least $k+1$ corners altogether.

On the other hand, given a feasible 3-coloring of $X$ by colors 2,4,6, a representation by $k$-gons can be achieved by placing the polygons $R_{a_{x}}, x \in X$ around the circle such that polygons corresponding to vertices of color $i$ are placed within the segment determined by the chord $R_{u_{i}}, i=2,4,6$. Each $c$ vertex can then be represented by 
a $k$-gon with one corner in each segment determined by $R_{u_{i}}, i=2,4,6, \ldots, 2 k$. An illustrative example is in Figure 6, where a triangle representing a triple $T=\{x, y, z\}$ is marked. For the sake of simplicity, we only illustrate the case $k=3$, and the auxiliary polygons $R_{b_{x}}$ are not shown (drawn with invisible ink).

Along the same lines it is seen that $\operatorname{cmp}\left(G_{\mathcal{T}}\right) \leq k+1$ if $(X, \mathcal{T})$ allows a 2-coloring without monochromatic triples.

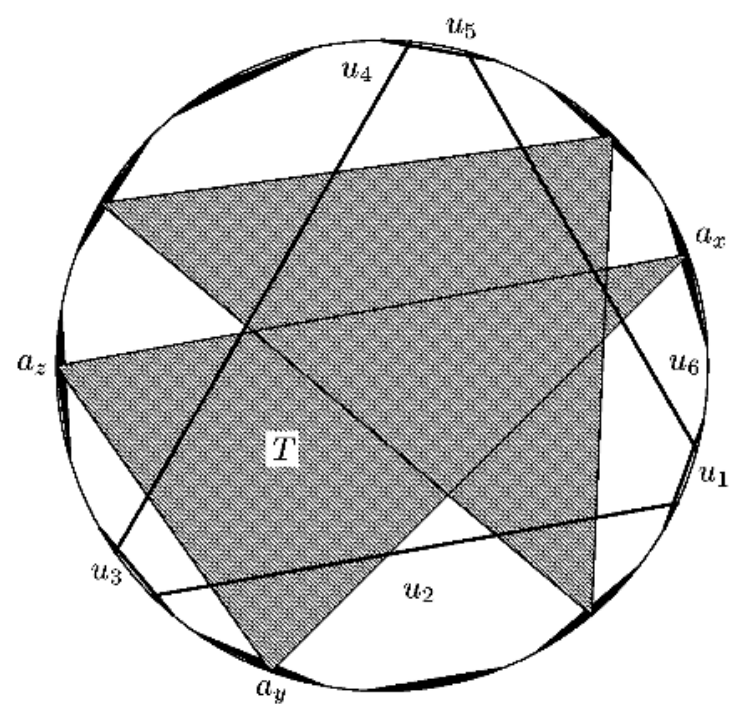

Fig. 6. Illustration to the NP-completeness proof.

One may ask for what function $f(n)$ of $n$ it is still hard to decide if $\operatorname{cmp}(G) \leq f(n)$ for an input polygon-circle graph $G$ on $n$ vertices (since we do not trust the polynomiality of polygon-circle graph recognition, we need to assume that the input graph comes with the promise of being a polygon-circle one). As we have seen in previous sections, $f(n)$ must be somewhat smaller than $n$ so that we might expect NP-hardness. By a closer examination of the proof of Theorem 2 we see that we can get close to $\frac{n}{2}$ :

Theorem 6. For every rational $c<\frac{1}{2}$, it is NP-complete to decide whether $\operatorname{cmp}(G) \leq$ cn for an input graph $G$ on $n$ vertices.

Proof. Consider the previous construction of $G_{\mathcal{T}}$. If we start the reduction from 3-edge coloring of a graph with $2 m$ vertices and $3 m$ edges, we get

$$
n=|V|=2 k+2|X|+|\mathcal{T}|=2 k+8 m .
$$


Hence setting $n=\frac{8 m}{1-2 c}$ (if $1-2 c>0$ is rational, we may start with sufficiently many copies of the cubic graph to make $n$ an integer) we get

$$
2 k=n-8 m=8 m\left(\frac{1}{1-2 c}-1\right)=2 c n
$$

and hence our previous proof shows that deciding

$$
\operatorname{cmp}\left(G_{\mathcal{T}}\right) \leq k=c n
$$

is NP-complete.

\section{Conclusion}

Regardless of whether recognition of polygon-circle graphs turns out polynomial or NPcomplete (if dichotomy is expected), our results about NP-hardness of polygon-circle graphs of bounded complicacy will be an interesting counterpart to the complexity of recognition of the whole class. We do hope that our paper will revitalize interest in the polygon-circle recognition problem as well.

\section{References}

1. A. Bouchet: Circle graph obstructions, J. Comb. Theory, Ser. B 60, No.1 (1994) 107-144

2. E.M. Eschen, J.P. Spinrad: An $O\left(n^{2}\right)$ algorithm for circular-arc graph recognition, in: Proceedings 4th ACM-SIAM Symposium on Discrete Algorithms, (1993), 128-137

3. H. de Frayssiex: A characterization of circle graphs, Eur. J. Comb. 5 (1984) 223-238

4. P.C. Gilmore, A.J. Hoffman: A characterization of comparability graphs and of interval graphs, Can. J. Math. 16 (1964) 539-548

5. A. Gyarfás: Problems from the world surrounding perfect graphs, Zastosow. Mat. 19, No.3/4 (1987) 413-441

6. I. Holyer: The NP-completeness of edge-coloring, SIAM J. Comput. 10 (1981) 713-717

7. M. Koebe: On a New Class of Intersection Graphs, Proceedings of the Fourth Czechoslovak Symposium on Combinatorics Prachatice, 141-143, 1990

8. J. Kratochvíl: String graphs II. Recognizing string graphs is NP-hard, J. Combin. Theory Ser. B, 52 (1991) 67-78

9. J. Kratochvíl, A. Kostochka: Covering and coloring polygon-circle graphs, Discrete Math. 163 (1997) 29-9-305

10. J. Kratochvíl, A. Lubiw, J. Nešetřil: Noncrossing subgraphs of topological layouts, SIAM J. Discrete Math. 4 (1991) 223-244

11. J. Kratochvíl, J. Matoušek: String graphs requiring exponential representations, J. Combin. Theory Ser. B 53 (1991) 1-4

12. J.Pach, G.Tóth: Recognizing string graphs is decidable, In: Graph Drawing (P. Mutzel, M. Jünger and S. Leipert, eds.), Proceedings 9th International Symposium GD 2001, Vienna 2001, Lecture Notes in Computer Science 2265, Springer Verlag Berlin Heidelberg 2002, pp. 247-260

13. J.P. Spinrad: Recognition of Circle Graphs, Journal of Algorithms 16 (1), 264-282, 1994

14. J.P. Spinrad: http://www . vuse .vanderbilt.edu/ ${ }^{\sim}$ spin/open .html 
15. M. Schaefer, D. Stefankovič: Decidability of string graphs, In: STOC 2001, Proceedings 33rd Annual ACM Symposium on Theory of Computing, Greece 2001, pp. 241-246

16. M. Schaefer, E.Sedgwick and D. Stefankovič: Recognizing string graphs in NP, In: STOC 2002, Proceedings 34th Annual ACM Symposium on Theory of Computing

17. A.C. Tucker: An efficient test for circular-arc graphs, SIAM J. Comput. 9 (1980) 1-24 